DOI: https://doi.org/10.31992/0869-3617-2019-28-11-69-77

\title{
Современная трактовка явления периодичности
}

Фадеев Герман Николаевич - А-р пед. наук, проф., кафедра химии. E-mail: gerfad@mail.ru Иебедев Юрий Александрович - канд. техн. наук, доцент, кафедра химии. E-mail: ruthenium1@yandex.ru

Авуличанская Наталья Николаевна - А-р пед. наук, проф., кафедра химии. E-mail: nnikdv@gmail.com

Московский государственный технический университет им. Н.Э. Баумана, Москва, Россия Aдрес: 107005 , г. Москва, 2-я Бауманская улица, 5

Аннотация. Представленнал работа посвящена анализу современного этапа развития учения о периодичности свойсть химических әлементов как фундаментального аспекта естественнонаучной картины мира. Изучение явления периодичности свойств химических элементов и его осознание научным сообществом давно выпили за рамки одной науки. Сегодня, после создания двух квантовьцх теорий электронного строения атома, столетнего опьта исследований химических элементов и экспериментального открытия антиводорода эта проблема далеко не исчерпана. Обобщение теоретических и экспериментальных исследовании квантово-механических аспектов химических свойств вещества и первых результатов изучения антивещества позволили выдвинуть гипотезу о существовании новой фундаментальной характеристики химического элемента и дать адекватную современнымм представлениям формулировку Периодического закона.

Ключевые слова: Периодическая таблииа, электронное строение атома, менделеевское число, Периодическии закон, современная формулировка, антиматерия

Аля иитирования: ФадееВ Г.Н., Аебедев Ю.А., Авуличанская Н.Н. Современная трактовка явления периодичности // Высшее образование в России. 2019. Т. 28. № 11. С. 69-77.

DOI: https://doi.org/10.31992/0869-3617-2019-28-11-69-77

\section{Введение}

K сегодняшнему времени исследование периодичности свойств химических элементов обрело черты универсального знания. По образному выражению академика С.И. Вольфковича, «закон является открытием взаимосвязи всех атомов мироздания» [1, c. 4]. Периодическая система химических элементов теперь включает в себя 118 «атомов мироздания»- индивидуальных химических элементов. Однако выяснение сути явления периодичности до сих пор остаётся чрезвычайно сложной научной проблемой, которая с открытием антивещества приобрела принципиально новый мировоззренческий аспект. При создании Амитрием
Ивановичем Менделеевым курса химии для студентов Санкт-Петербургского университета перед ним как лектором возникла проблема логичного изложения материала на единой концептуальной основе. Аля химии, как и для любой другой науки, важен принции классификации, по которому происходит рассмотрение и отбор необходимых отдельных структурных элементов при создании единого целого. Такой принцип классификации химических элементов ещё до рождения Амитрия Ивановича (1834 г.) был найден немецким учёным И. Аёберейнером, который на основании сходства свойств хи- 


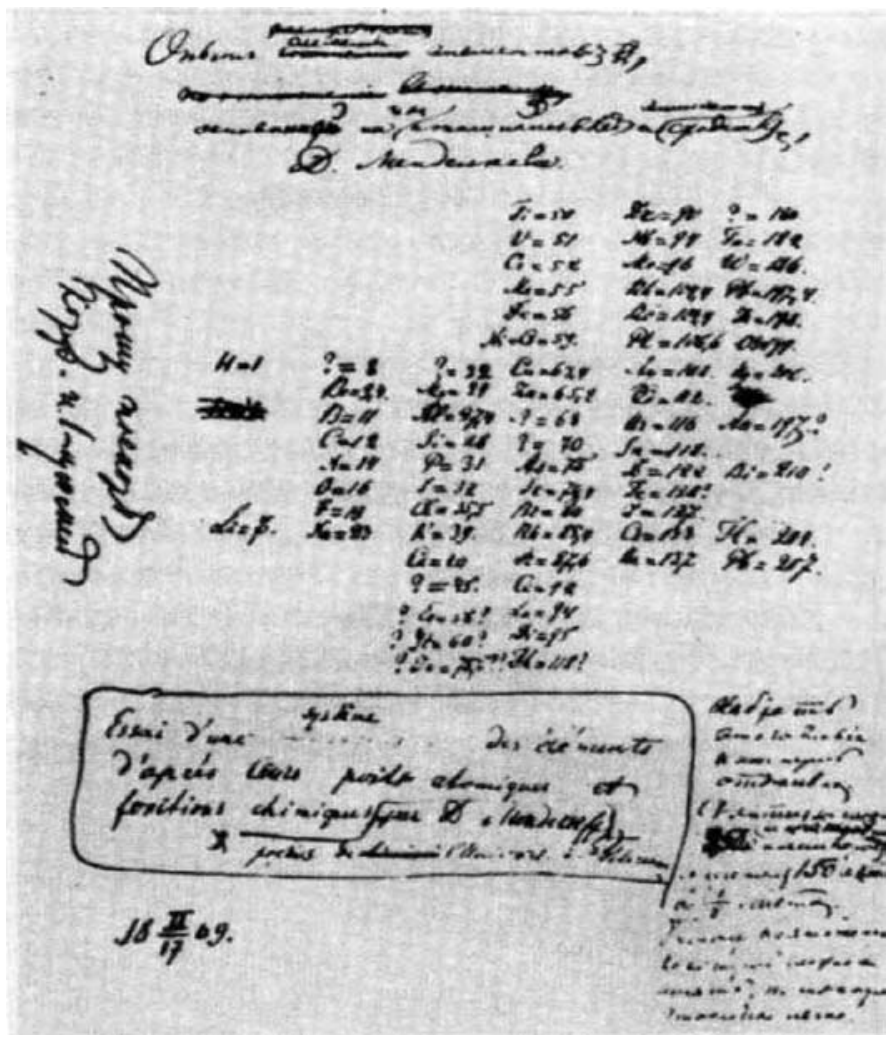

Фотография первого варианта периодической таблицы химических элементов (внизу слева дата - 17.II.1869.) (http://www.thingshistory.com)

мических элементов расположил их триадами по возрастанию атомного веса. В 1817 г. он опубликовал сведения о первой триаде, а к 1829 г. их стало пять. Они включали в себя пятнадцать элементов - менее половины из известных к тому времени. Самое же главное - обнаружился стержневой принцип: $3 a$ висимость свойсть химических элементов от их атомной массы. В триадах атомная масса среднего элемента была среднеарифметической величиной из атомных масс двух крайних элементов. Автор «триад», однако, не решился распространить обнаруженную им закономерность на другие известные к тому времени элементы. В своих работах по классификации химических элементов А.И. Менделеев дал пример не только әмпирического, но и философского обоснования закона. Можно с уверенностью утверждать, что научное и философское значение Периодической системы и Периодического закона А.И. Менделеева в продолжение полуторавекового их существования не поколеблено ни одним из революционных научных приобретений в области строения вещества.

В 1869 г. - к моменту появления первого варианта Периодической системы - понятие «атом» не имело физического обоснования, являясь философской абстракцией. По представлениям естественнонаучных разделов естествознания того времени, таких как физика и химия, атом считался неделимой частицей. На эти представления опирался и Менделеев. Однако открытие электрона, входящего в состав атома (1895-1897 гг.), так же как и обнаруженное Э. Резерфордом через пятнадцать лет, в 1911 г., сложное строение атома, нисколько не умалили значение 
менделеевского открытия. Эти достижения мировой науки, наоборот, укрепили величие Периодического закона. Существенные перемены в понимании Периодического закона, вызвавшие изменение его формулировки, внесли опыты английского физика Г. Мозли. Проведённый им в 1913 г. анализ экспериментально полученных рентгеновских спектров химических элементов показал: атомный (порядковый) номер химического элемента в табличе Менделеева являетсл фундаментальной физической характеристикой этого атома. Г. Мозли предположил, что такой характеристикой является заряд ядра.

В 1920 г. Аж. Чедвик непосредственным измерением рассеяния $\alpha$-частиц на ядрах платины, серебра и меди определил заряды их ядер и подтвердил равенство этих зарядов порядковым номерам исследованных элементов. Усвоение результатов описанных открытий привело к той формулировке Периодического закона, которая используется до сего времени в энциклопедиях [2, с. 985; 3, c. 432] и учебниках для высшей школы [4, с. 141; 5, с. 48; 6, с. 96]: «Свойства химических элементов... находятся В периодической зависимости от зарядов атомньх ядер». В приведённой формулировке нет обълснения сути лвления периодичности. Выяснение причин периодичности свойств химических элементов, начавшееся в двадцатых годах прошлого столетия, потребовало нескольких десятилетий работы общемировой научной мысли, создания двух теоретических представлений: квантово-корпускулярной теории Резерфорда - Бора и квантововолновой теории Шредингера - Гейзенберга, - чтобы количественно описать состояние электронов в многоэлектронных атомах.

\section{Хроника открытия}

Появление первой менделеевской периодической таблицы и оглашение лежащего в её основе закона оказались разнесёнными по времени, поэтому так важно уяснить хронологию этого события. Историки химии подсчитали, что А.И. Менделеев был 53-м исследователем, занимавшимся систематизацией химических элементов. И всё же именно он признан первооткрыъателем закона природь, на котором строится Периодическая система химических элементов. Успех А.И. Менделеева стал возможен потому, что он рассматривал периодичность, опираясь не на одну, как делали его предшественники, а на две основы систематизации. В соответствии с разработанным им «методом атомной аналогии» А.И. Менделеев брал в рассмотрение не только атомную массу элементов, но и аналогию их химических свойств. Этот факт отражён в самом названии Первой таблицы Периодической системы химических элементов, которая появилась 17 февраля 1869 г.: «Опыт системы элементов, основанной на их атомном весе и химическом сходстве». 22 февраля 1869 г. Амитрий Иванович закончил статью под названием «Соотношение свойств с атомным весом элементов». Этот труд от имени А.И. Менделеева был 6 марта 1869 г. представлен секретарём Русского химического общества профессором Н.А. Меншуткиным на заседании этого научного собрания и напечатан в майском номере журнала этого общества.

Высокая интенсивность работы над статьёй и небывало короткий срок её публикации свидетельствуют об осознании исключительной важности события. В статье А.И. Менделеев делал важнейший вывод: «Элементы, расположенные по величине их атомного веса, представляют явственную периодичность свойств». Так во всемирном химическом словаре появился новый термин - периодичность свойств химических элементов, однако открытие самого закона было ещё впереди. Этому новому термину ещё предстояло войти в формулировку закона, на обоснование которого Амитрию Ивановичу потребовалось полтора года напряжённой работы.

Открытый А.И. Менделеевым закон природы, названный им «законом периодичности», впервые сформулирован в 1870 г. 
в учебнике «Основы химии»: «Свойства простых тел, также формы и свойства соединений элементов, находятся В периодической зависимости от величины атомных Весов элементов" - и уточнён в последнем прижизненном издании $[7$, с. 80]. Убеждённый в справедливости созданной им Периодической системы, А.И. Менделеев, во втором варианте Периодической таблицы предсказывает существование уже не четырёх (как сделано им в первом варианте), а двенадцати химических элементов. Некоторые из них учёный описал так подробно, что заставил пересматривать результаты экспериментальных измерений. Глубокое и всестороннее обоснование Периодического закона и Периодической системы изложено в 15 наиболее известных трудах Амитрия Ивановича. Самым выдающимся своим научным произведением А.И. Менделеев считал учебник «Основы химии», выдержавший более десяти изданий, из которых последнее прижизненное вышло в свет в 1906 г. Эта книга рекомендовалась в качестве учебника для химических факультетов отечественных университетов вплоть до середины XX века. Химический этап развития учения о периодичности, начавшийся 17 февраля 1869 г., Алился до 1911 года.

\section{ЯАерный периоА развития}

Следующий период изучения явления периодичности, который можно назвать ядерныıм, наступил после выявления Э. Резерфордом в 1911 г. факта строения атомов химических элементов. Атом, считавшийся прежде бесструктурныљм, оказался иерархически сложным образованием! Аля установления этого факта было использовано недавно открытое А. Беккерелем явление радиоактивного распада атомов, подробно изученное самим Резерфордом, открывшим три вида продуктов распада: $\alpha-, \beta-, \gamma$-излучения. При экспериментальном измерении отклонения от прямолинейного пути $\alpha$-частиц, проходящих сквозь золотую фольгу, обнаружился потрясающий факт: атом имеет тяжёлое положи- тельно заряженное ядро в окружении лёгких отрицательно заряженных электронов.

Открытие электронной компоненты атомов химических элементов и прямой связи её структуры с химическими свойствами элементов приоткрыло логику связи заряда атомного ядра (нуклида), определяющего количество электронов в атоме, с химическим поведением всей нуклидо-электронной системы. Аля детализации этой логики до возможности выявления количественных закономерностей химического поведения атомов элементов потребовалось создание квантово-механической модели атома. Эту задачу в общем виде удалось решить Н. Бору.

Квантово-механическое объяснение периодичности, выдвинутое его школой и сводящееся к констатации корреляции между структурой внешних электронных оболочек и общим характером химических свойств атома, явилось весьма продуктивным для прагматичного исподьзования явления периодичности.

Однако оно не внесло окончательной ясности в понимание сути этого явления. Оставим в стороне обсуждение формальных «шероховатостей» этого объяснения, вызванных многочисленными отклонениями структур электронных оболочек от «идеальной» последовательности: $1 \mathrm{~s}<2 \mathrm{~s}<2 \mathrm{p}<3 \mathrm{~s}$ $<3 \mathrm{p}<3 \mathrm{~d}<4 \mathrm{~s}<4 \mathrm{p}<4 \mathrm{~d}<4 \mathrm{f}<\ldots$.., и нарушениями последовательности заполнения орбиталей электронами (различные «провалы», нарушающие правило Гунда). Скажем о главном: объяснение не раскрывает природу химической связи и конкретные виды функций периодичности свойств атомов: потенциалов ионизации, сродства к электрону, электроотрицательности и других.

\section{Структурность взаимосвязанных систем} фундаментальных частиц как первооснова явления химической периодичности

Особенно важным для уяснения причин периодичности свойств химических элементов является открытие и синтез антиэлементов, в первую очередь - антиводорода. 
Обсудим основные результаты этих исследований. После предсказания Аираком в 1928 г. существования антиматерии, первая античастица - позитрон - была открыта в 1932 г. американским физиком К.А. Андерсеном в космических лучах при помощи камеры Вильсона, помещённой в магнитное поле. Автор удостоен Нобелевской премии по физике за 1936 г. Нуклид - антипротон - открыт в 1955 г. на ускорителе протонов в Калифорнийском университете в Беркли.

15 сентября 1995 г. группа физиков под началом В. Олерта в ЦЕРНе (Швейцария) синтезировала первые атомы антиводорода. В настоящее время изучаются уже «газовые облачка», состоящие из 50000 атомов антиводорода. Эти атомы, в отличие от атомов водорода, имеют заряд ядра не +1 , а -1 , их энергетические орбитали заняты не электронами, а позитронами. Химические свойства атомов антиводорода пока только начинают изучаться [8]. В 2016 г. в ЦЕРНе большой группой исследователей (в том числе и российских) установлено, что энергия перехода из состояния $1 s$ в $2 s$ у этих атомов совпадает с энергией такого же перехода у атомов «обычного водорода» с точностью до $\sim 10^{-10}$ [9]. Это даёт достаточное основание утвержАать, что водород и антиводород - один $и$ тот же химический элемент, обладаюший двумя типами зарядов ядер атома.

Из этого факта следует чрезвычайно важный вывод: формулировка Периодического закона как зависимости периодичности свойств химических элементов от заряда ядер теряет смьсл, поскольку заряд ядра неоднозначно привязывает нуклидо-лептонную (в частности, для «обычных элементов» нуклидо-электронную) группировку к определённому месту в Периодической таблице. Факт существования антиматерии делает привязку атомного номера к значению заряда ядра несостоятельной. Эксперимент в ЦЕРНе подтвердил «физическую возможность» взаимного преобразования миров материи и антиматерии. С физической точки зрения симметричность этих миров со-

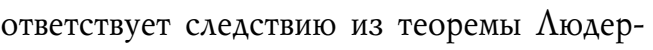
са - Паули (или СРТ-теоремы) [10; 11], которая гласит: «При зеркальном отражении пространственных координат, изменении знака электрического заряда и обрашении направления течения времени физические свойства вещества и антивещества остаются строго тождественными».

Не вдаваясь в детали физических представлений о свойствах материи и антиматерии, рассмотрим, какое отношение это имеет к химии и Периодическому закону. Прежде всего, отметим, что антиматерия $b$ форме нуклидов химических элементов - это уже экспериментальный факт. Известны ионы антидейтерия и изотопов антигелия. Самое тяжёлое на настоящий момент «антиядро» получено в лаборатории Брукхевейна: при столкновениях пучков ионов золота появились ионы антигелия-4 (aнmu- $\left.{ }^{4} \mathrm{He}^{+2}\right)$ [12]. Если мы допускаем, что $\beta^{-}$-распад и $\beta^{+}$распад - это разные по своим параметрам процессы в мире материи и антиматерии, то это означает, что и другие свойства атомов, подверженных этим видам распада, могут быть разными. А в числе этих других свойств могут быть и химические свойства, поскольку свойства ядер влияют на энергетику орбиталей (электронных «у нас» и позитронных - в антимирах). Следовательно, заряд ядра нельзя полностью отождествлять с порядковьм номером әлемента, так как он является более частной его характеристикой.

Заряд ядра имеет знак: положительный - для обычных элементов и отрицательный - для антиэлементов. «Порядковый номер элемента»- знака заряда не имеет. Следовательно, если справедлива СРТ-теорема, таблица антиэлементов, которые будут синтезированы (или открыты где-либо во Вселенной), будет иметь точно такой же вид, как и привычная нам Периодическая таблица Менделеева. Сегодня, однако, настоящего нуклидо-позитронного антивещества в «химически связанной форме» (т.е. в форме молекул или молекулярных ионов) ещё не получено. Поэтому 
существует ли химическая симметрия «миров» и «антимиров»- вопрос на сегодня открытый. Есть основания предположить, что «незначительные отличия» ядерных свойств анти-элементов могут привести к более точной балансировке сил их взаимодействия с позитронными оболочками, и в Периодической таблице антиэлементов, возможно, исчезнут те исключения из правила «Маделунга - Клечковского» [13; 14, c. 603], которые наблюдаются в существующей таблице элементов. Идея эквивалентности «физичности» и «математичности», хотя не выраженная явно, очевидно, была близка мироощущению А.И. Менделеева, который в последней прижизненной версии Периодического закона формулирует её так: "Свойства простых тел, также формы и свойства соединений элементов, находятся в периодической зависимости (или, выражаясь алгебраически, образуют периодическую функцию) от величинь атомных весов элементов » [15, с. 254].

Как следует из предшествующего изложения проблемы, химическая периодичность является функцией некоторой величины, которая имеет фундаментальное значение и математически соответствует натуральному ряду чисел. Сегодня в качестве такой величинь принято использовать заряд ядра. Это допущение, однако, кроме обсуждённого выше физического возражения против такого отождествления в связи с существованием антиматерии, содержит в себе логическую несообразность. Заряд ядра - это размерная величина, имеющая различные значения в зависимости от принятых единиц измерения. В системе СИ единицей измерения электрического заряда является кулон. В таком случае изменение заряда ядер при переходе от элемента к элементу в Периодической таблице соответствует изменению заряда на величину шага в $1,6 \cdot 10^{-19} \mathrm{~K}$. Аля придания порядковому номеру элемента правильного физического смысла в формулировке Периодического закона необходимо указывать, что под зарядом ядра имеется в виду приведённая величина, нормированная на единичный электрический заряд. Такое дополнение формулировки фундаментального химического закона затрудняет восприятие и привносит в его формулировку несущественные частности. Вместе с тем они снижают ранг его фундаментальности.

Фундаментальная характеристика химического элемента - менделеевское число

Указанные методологические трудности с определением аргумента функиии химической периодичности сегодня преодолеваются введением понятия «порядковый номер» химического элемента. Однако это не разрешает всех логических проблем. Прежде всего, само понятие «порядковый номер» подразумевает существование критерия, по которому производится упорядочение. В химии таких критериев может быть множество: место элемента в $A$-последовательности орбиталей; электроотрицательность атомов; первый потенциал ионизации; сродство к электрону; электродный потенциал и т.п.

Во избежание путаницы, возникающей с термином «порядковый номер» в связи с разнообразием химических характеристик элементов, предлагается следующее. Отмечая основополагающий вклад А.И. Менделеева в открытии смысла понятия «химический элемент», заменить термин «порядковыий номер химического элемента" фундаментальной характеристикой - «менделеевское иисло» $(M \varkappa)$. Введение данного термина в год 150-летия Периодической таблицы включит имя первооткрывателя Периодического закона в число мировых констант, ещё более укрепит авторитет А.И. Менделеева и будет являться свидетельством международного признания заслуг нашего великого соотечественника.

Как ясно из предыдущего изложения, $x u$ мическая периодичность является функиией менделеевского числа - величинь, имеющей фундаментальное значение и математически соответствуюшал натуральному ряду чисел. Заряд ядра, по сравнению с менделеевским числом химического элемента, 
является более частной характеристикой. Физический смысл менделеевского числа в том, что оно представляется квантовым числом, характеризуюшим качественные скачки изменения химических свойств элементов. Само понятие «химические свойства» обретает с введением менделеевского числа количественную характеристику. В таком случае смысл явления периодичности раскрывается как связь количественных характеристик «химичности» әлемента с «физичностью» структуры его элементарной единицы - атома.

Итак, что можно сказать об исследовании явления периодичности свойств химических элементов сегодня - в год 150-летия Периодической таблицы А.И. Менделеева? Подводя итог приведённым рассуждениям, можно констатировать следующее. Явление периодичности, опираясь на новейшие достижения экспериментальной и теоретической химии и физики, играет интегрирующую роль в процессе естественнонаучного познания. Современное его состояние открывает принципиально новые стратегические горизонты перед теоретической и прикладной химией. Целесообразно было бы обратиться к международным организациям химиков с предложением внести в формулировку Периодического закона изменения, касающиеся роли электронной оболочки атомов. Периодический закон А.И. Менделеева может быть сформулирован следующим образом: «Свойства химических элементов в нуклидолептонных формах атомов и ионов, а также формы и свойства соединений этих элементов, находятся в периодической зависимости от менделеевского числа, определяющего структуру и основные свойства химического проявления их электронной конфигурации».

Аля учебных целей эту формулировку можно упростить: «Свойства химических элементов, а также формь и свойства соединений этих элементов находятся в периодической зависимости от менделеевского числа, определяюшего структуру электронной конфигурачии атомов ».
Смысл такого нововведения состоит не только в том, чтобы отдать дань прошлому химии, но и в том, чтобы на его основе строить планы будущего развития учения о периодичности. Вспомним приведённую выше менделеевскую формулировку ПериоАического закона 1906 г., в которой он провидчески ввёл «алгебраическую функиию» в качестве коррелята между физическими свойствами «атомный вес» и «химические свойства». Вдумаемся: в этой формулировке физические свойства и химические взаимодействия логически эквивалентны некоторой периодической «алгебраической фрункиии»! Применительно к обсуждаемой теме современной формулировки Периодического закона эти идеи порождают вопросы об универсализме как самого химического взаимодействия атомов, так и его проявлений в рамках этого закона.

\section{Митература}

1. Вольфкович С.И. Вводная статья // Сто лет периодического закона химических элементов. Сб. докл. Х юбил. Менделеевского съезда. М.: Наука, 1969.

2. Советский энциклопедический словарь. М.: Большая Советская энциклопедия, 1983.

3. Химия // Большой энциклопедический словарь. М.: Большая Росс. энциклопедия, 2000.

4. Тамм М.Е., Третьяков Ю.А. Неорганическая химия: в 3 т. Т. 1. Физико-химические основы неорганической химии: Учебник для студентов высших учебных заведений. М.: Академия, 2000.

5. Росин И.В., Томина А.А. Общая и неорганическая химия. Современный курс: Учебное пособие для бакалавров и специалистов. М.: Юрайт, 2012. 1338 с.

6. Аебедев Ю.А., Фадеев Г.Н., Голубев А.М., Шаповал B.M. Химия: Учебник для академического бакалавриата. М.: Юрайт. 2016.

7. МенделееВ А. Основы химии. Изд.13-е (5-е посмертное). М.- $\Lambda .:$ Гос. науч.-техн. изд-во химической литературы, 1947. Т. 2.

8. Грушина А. Антиводород: новая эра экспериментов с антиматерией // Наука и жизнь. 2017. № 1. URL: https://www.nkj.ru/archive/ articles/30451/ 
9. Abmad M., Alves B.X.R., Baker C. J., Bertsche W., ButlerE., Capra A, Carruth C., Cesar C.L., Charlton M., Cohen S., Collister R., Eriksson S., Evans A., Evetts N., Fajans J., Friesen T., Fujiwara M.C., Gill D.R., Gutierrez A., Hangst J.S., Hardy W.N., Hayden M.E., Isaac C.A., Ishida A., Johnson M.A., Jones S.A., Jonsell S., Kurchaninov L., Madsen N., Mathers M., Maxwell D., McKenna J.T.K., Menary S., Michan J.M., Momose T., Munich J.J., Nolan P., Olchanski K., Olin A., Pusa P., Rasmussen C.Ø., Robicheaux F., Sacramento R.L., Sameed M., Sarid E., Silveira D.M., Stracka S., Stutter G., So C., Tharp T.D., Thompson J.E., Thompson R.I., van der Werf D.P., Wurtele J.S. Observation of the 1S-2S transition in trapped antihydrogen // Nature. 2017, January, Vol. 541, pp. 506-510. URL: https://www.nature.com/ articles/nature21040/

10. Luders $G$. On the Equivalence of Invariance under Time Reversal and under Particle-Antiparticle Conjugation for Relativistic Field Theories //
Kgl. Danske Videnskab. Selskab, Mat-Fys. Medd. 1954. Vol. 28. No. 5.

11. Pauli $W$. Niels Bohr and the Development of Physics. N.Y., 1955.

12. Walsh Karen McNulty, Genzer Peter. RHIC Physicists Nab New Record for Heaviest Antimatter // Brookhaven National Laboratory. 2011. April 24.

13. Madelung E. Die mathematischen Hilfsmittel des Physikers, Springer Verlag, Berlin, 1936.

14. Клечковский B.M. $(n+l)$-группы в последовательном заполнении электронных конфигураций атомов // Аоклады АН СССР. 1951. Т. 80.

15. Менделеев А.И. Основы химии. 8-е издание, вновь исправленное и дополненное. С.-Петербург, Типо-литография М.П. Фроловой, 1906.

Статья поступила в редакцию 05.03.19 Принята к публикачии 02.10.19

\section{Modern Interpretation of Periodic Phenomenon}

German N.Fadeev - Dr. Sci. (Education), Prof., Department of chemistry, e-mail: gerfad@mail.ru

Yuri A. Lebedev - Cand. Sci. (Engineering), Assoc. Prof., Department of chemistry, e-mail: ruthenium1@yandex.ru

Natalya N. Dvulichanskaya - Dr. Sci. (Education), Prof., Department of chemistry, e-mail: nikdv@gmail.com

Bauman Moscow State Technical University, Moscow, Russia

Address: 5, 2nd Baumanskaya str., 107005, Moscow, Russian Federation

Abstract. The presented work is devoted to the analysis of the modern stage of development of the theory periodic properties of chemical elements as a fundamental aspect of scientific worldpicture. Studying of the phenomenon of periodic properties of chemical elements has gone far beyond chemistry. It should be taken into account that even today, after the creation of two quantum theories of the electronic structure of the atom, after a century of experience in the study of chemical elements and the experimental discovery of antihydrogen, this problem is far from being exhausted. The generalization of theoretical and experimental studies of the quantum-mechanical aspects of the chemical properties of matter, the first results of the study of antimatter made it possible to set a hypothesis of the existence of a new fundamental characteristic of chemical element and to formulate the Periodic Law adequately - in keeping with modern concepts.

Keywords: Periodic table, Periodic Law, electronic structure of the atom, Mendeleev number, antimatter

Cite as: Fadeev, G.N., Lebedev, Yu.A., Dvulichanskaya, N.N. (2019). Modern Interpretation of the Periodic Phenomenon. Vysshee obrazovanie v Rossii= Higher Education in Russia. Vol. 28, no. 11, pp. 69-77. (In Russ., abstract in Eng.)

DOI: https://doi.org/10.31992/0869-3617-2019-28-11-69-77 


\section{References}

1. Wolfkovich, S.I. (1969). Introductory article. In: Sto let periodicheskogo zakona kbimicheskikb elementov [One Hundred Years of the Periodic Law of Chemical Elements: Proc. X Mendeleev Congress]. Moscow: Nauka Publ. (In Russ.)

2. Sovetskiy entsiklopedicheskiy slovar' (1983). [Soviet Encyclopedic Dictionary]. Moscow: Bol'shaya Sovetskaya entsiklopediya Publ. (In Russ.)

3. Khimiya (2000). [Chemistry]. In: Bol'shoy entsiklopedicbeskiy slovar' [Great Encyclopedic Dictionary], Moscow: Bol'shaya Rossiiskaya entsiklopediya Publ. (In Russ.)

4. Tamm, M.E., Tret'yakov, Yu.D. (2000). Neorganicheskaya kbimiya: $v 3 t$. [Inorganic Chemistry: in 3 Volumes: Textbook for higher education institution students]. Vol. 1. Physico-Chemical Bases of Inorganic Chemistry. Moscow: Academiya Publ. (In Russ.)

5. Rosin, I.V., Tomina, L.D. (2012). Obshchaya i neorganicheskaya kbimiya. Sovremenniy kurs: Uchebnoe posobie dlya bakalavrov $i$ spetsialistov [General and Inorganic Chemistry. Modern course: Textbook for Bachelors and Specialists]. Moscow: Yurait Publ. (In Russ.)

6. Lebedev, Yu. A., Fadeev, G.N., Golubev, A.M., Shapoval, V.M. (2016). Khimiya: Uchebnik dlya akademicheskogo bakalavriata [Chemistry: Textbook for Academic Baccalaureate]. Moscow: Yurait Publ. (In Russ.)

7. Mendeleev, D. (1947). Osnovy khimii. Izd.13-e (5-e posmertnoe) [Fundamentals of Chemistry.13th Ed. (5th posthumous)]. Vol. 2. Moscow - Leningrad: State Scientific and Technical Publishing House of Chemical Literature. (In Russ.)

8. Grushina, A. (2017). Antihydrogen: A New Era of Experiments with Antimatter. Nauka i Zbizn' = Science and Life. No. 1. Available at: https://www.nkj.ru/archive/articles/30451/ (In Russ.)

9. Ahmadi, M., Alves, B.X.R., Baker, C.J., Bertsche, W., Butler, E., Capra, A, Carruth, C., Cesar, C.L., Charlton, M., Cohen, S., Collister, R., Eriksson, S., Evans, A., Evetts, N., Fajans, J., Friesen, T., Fujiwara, M.C., Gill, D.R., Gutierrez, A., Hangst, J.S., Hardy, W.N., Hayden, M.E., Isaac, C.A., Ishida, A., Johnson, M.A., Jones, S.A, Jonsell, S., Kurchaninov, L., Madsen, N., Mathers, M., Maxwell, D., McKenna, J.T.K., Menary, S., Michan. J.M., Momose, T., Munich, J.J., Nolan, P., Olchanski, K., Olin, A., Pusa, P., Rasmussen, C.Ø., Robicheaux, F., Sacramento, R.L., Sameed, M., Sarid, E., Silveira, D.M., Stracka. S., Stutter. G., So, C., Tharp, T.D., Thompson, J.E., Thompson, R.I., van der Werf, DP, Wurtele, J.S. (2017). Observation of the 1S - 2S Transition in Trapped Antihydrogen. Nature, January. Vol. 541, pp. 506-510. Available at: https://www. nature.com/articles/nature21040/

10. Luders, G. (1954). On the Equivalence of the Invariance under Time Reversal and Under Particle-Antiparticle Conjugation for Relativistic Field Theories. Kgl. Danske Videnskab. Selskab, Mat-Fys. Medd. Vol. 28, no. 5.

11. Pauli, W. (1955). Niels Bohr and the Development of Physics. N.Y.

12. Karen McNulty Walsh, Genzer, Peter (2011). RHIC Physicists Nab New Record for Heaviest Antimatter. Brookbaven National Laboratory, April 24.

13. Madelung, E. (1936). Die mathematische Hilfsmittel des Physikers, Springer Verlag. Berlin.

14. Klechkovsky, V.M. (1951). (n + l) -Groups in the Sequential Filling of Electron Configurations of Atoms. Doklady Akademii nauk SSSR [Reports of the USSR Academy of Sciences]. Vol. 80. (In Russ.)

15. Mendeleev, D.I. (1906). Osnovy kbimii. 8-e izdanie, vnov' ispravlennoe i dopolnennoe [Basics of Chemistry. 8th Edition, Newly Revised and Augmented], St. Petersburg: Publ. by Tipo-lithography M.P. Frolova. (In Russ.) 